Dept. for Speech, Music and Hearing Quarterly Progress and Status Report

\title{
Perception of just noticeable time displacement of a tone presented in a metrical sequence at different tempos
}

Friberg, A. and Sundberg, J.

journal: STL-QPSR

volume: 34

number: $2-3$

year: $\quad 1993$

pages: $\quad 049-056$ 



\title{
PERCEPTION OF JUST NOTICEABLE TIME DISPLACEMENT OF A TONE PRESENTED IN A METRICAL SEQUENCE AT DIFFERENT TEMPOS
}

\author{
Anders Friberg and Johan Sundberg
}

\begin{abstract}
The JND for a perturbation of the timing of a tone appearing in a metrical sequence was examined in an experiment where 30 listeners of varied musical background were asked to adjust the timing of the fourth tone in a sequence of six fixed tones, such that they heard the sequence as perfectly regular. The tones were presented at a constant interonset time that was varied between $100 \mathrm{~ms}$ and $1000 \mathrm{~ms}$. The average JND was found to be about $10 \mathrm{~ms}$ for tones shorter than about $240 \mathrm{~ms}$ duration and about $5 \%$ of the duration for longer tones. Subjects' musical training did not appear to affect the se values.
\end{abstract}

\section{INTRODUCTION}

Small perturbations of the duration of tones in a musical performance is an important part of musical quality. The relevance of tone durations to music performance has been demonstrated in many previous investigations (Schaffer, 1981; Gabrielsson and Bengtsson, 1983; Clarke, 1982; Sloboda, 1983; Sundberg et al., 1991b; Todd, 1985; Repp, 1992a). However, the scientific interest have focused mainly on the analysis of music performances while the perceptual aspect has previously been somewhat neglected. Until now, investigations of the perception of time intervals have had a more basic psychoacoustic orientation. However, recently a widening of the horizon has been noted (Repp, 1992b; Hirsh et al., 1990; Monahan and Hirsh, 1990; Clarke 1989; Sundberg et al., 1991a).

In our ongoing research on musical performance, we have formulated rules which alter the duration of certain notes depending on the musical context (Friberg, 1991). In this work, it would be very helpful to define a rule, the effect of which remains perceptually the constant, when the tempo is varied. The problem is that musical excerpts are typically correlated with tempo. Therefore, a certain melody can not be transposed too much in tempo.

The present investigation is a first attempt to solve this problem by determining the just noticeable difference (JND) in duration in a very simple context.

An important question is whether these perturbations should be calculated as relative or absolute deviations from nominal durations. Relative deviations imply that the perturbation is in percent of the note's nominal duration, while absolute deviations mean that a fixed amount in milliseconds is added or subtracted.

A strong argument favour relative deviations; for example, the note values in music notation is built on the principle of doubling and halving. On the other hand, a per- 
fectly strict application of the doubling/halving principle does not seem likely; a percentual lengthening of a long note may add extra beats. In our performance rule system we currently use both strategies. Most rules are based on relative deviations, but some also use absolute deviations.

\section{EXPERIMENT}

To isolate the durational aspect of perception, minimising other possibly influential factors such as melody, rhythmic pattern, musical form etc., a rather simple context were chosen. The stimuli consisted of six notes played in succession, see Fig 1. All notes were equally spaced in time except the fourth note which could be moved back and forth relatively the other notes. For each trial, the subject was asked to adjust the fourth note until all notes appeared to be evenly spaced in time.

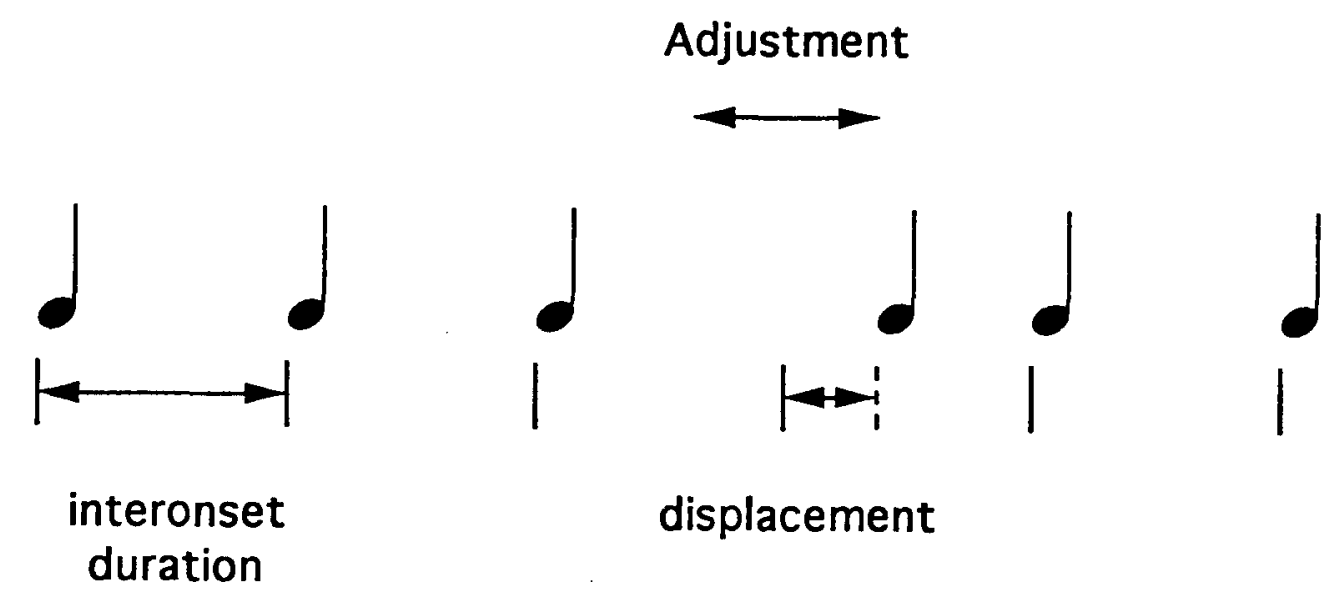

Fig. 1. The stimuli consisted of a sequence of six notes. All notes were fixed except the fourth, which could be moved back and forth in time relatively the others.

Nine interonset durations ranging between 100 and 1000 ms were used. Each duration occurred three times totalling 27 trials in the test. Initially three test trials were presented for reasons of practice. Information about the subjects' musical experience was collected, as well as the number of repeats they spent in each trial. The whole test took between 20 and 40 min. and was automatically run by a Macintosh II computer. The stimulus order and the initial displacement value were all randomly varied.

Standard MIDI equipment was used with the computer connected to a Yamaha FB01 synthesiser. The sound used had a guitar-like timbre with very fast attack ( 0.5 ms) and an slow, immediate decay. Due to the MIDI transmission the accuracy was rather modest. The variation of the timing was $2 \mathrm{~ms}$. This was considered acceptable and smaller than the expected results.

Most of the subjects were students at the Royal Institute of Technology studying engineering and taking the course in music acoustics. Around half of them were amateur musicians and one of them was a professional. There were totally 30 subjects in the test. The subjects were divided into two groups according to the length of their reported musical experience. It turned out that half of the subjects were musically more or less active, while the other half of the subjects lacked musical experience almost entirely. 


\section{RESULTS}

The mean of twice the absolute value of the subjects' error relative to mechanical regularity, henceforth the displacement, was chosen as an estimate of the JND:

$$
J N D=\operatorname{MEAN}\left(2 * \mid \text { displacement }_{i} \mid\right)
$$

The overall result is shown in Fig. 2, with the relative JND as a function of the interonset durations. The error bars indicate $95 \%$ confidence intervals. As can be seen, the JND curve is rather constant for durations higher than $250 \mathrm{~ms}$.

Fig. 2. The JND of duration in percent of the interonset duration as a function of interonset duration for the displacement of the fourth note in a six note sequence. The bars indicate $95 \%$ confidence intervals for each value.

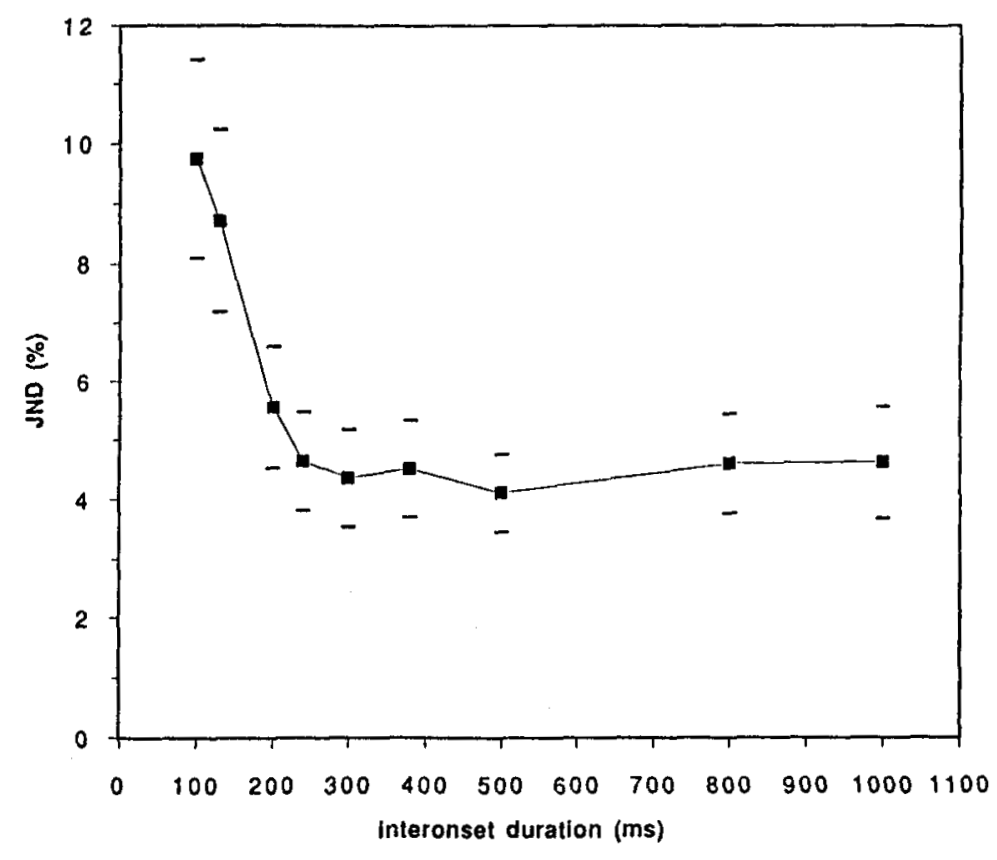

In Fig. 3, the same result is displayed with absolute rather than the relative JND. For durations shorter than $250 \mathrm{~ms}$ the absolute JND is almost constant. These results seem to suggest that two different processes are used for the perception of time intervals of this type.

Fig. 3. The JND of duration as a fixed amount in ms as a function of the interonset duration for the displacement of the fourth note in a six note sequence. The bars indicate $95 \%$ confidence intervals for each value.

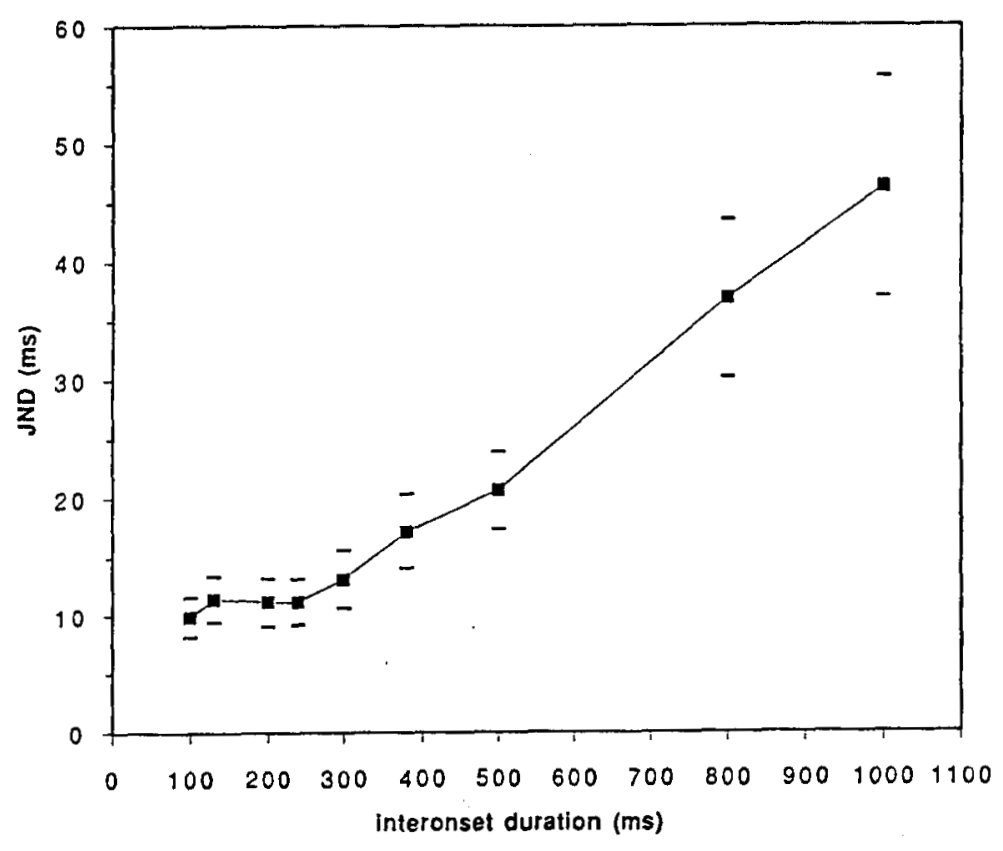


Somewhat surprisingly, no effect of musical experience was observed in this group of subjects; there were no significant differences between the two groups for any of the measured interonset durations.

Another parameter that the program stored was the number of repetitions the subject used in each trial. In Fig. 4 the mean values of this number are shown as a function of interonset duration. The number of repetitions can be assumed to reflect the difficulty of the task. Interestingly, the number of repetitions follow the JND curve quite closely. This indicates that the perceived difficulty of the task was dependent on the JND. There were no significant differences between the results from the musicians and the non-musicians in this regard.

Fig. 4. Mean of the subject's number of repetitions of each stimulus as a function of interonset duration.

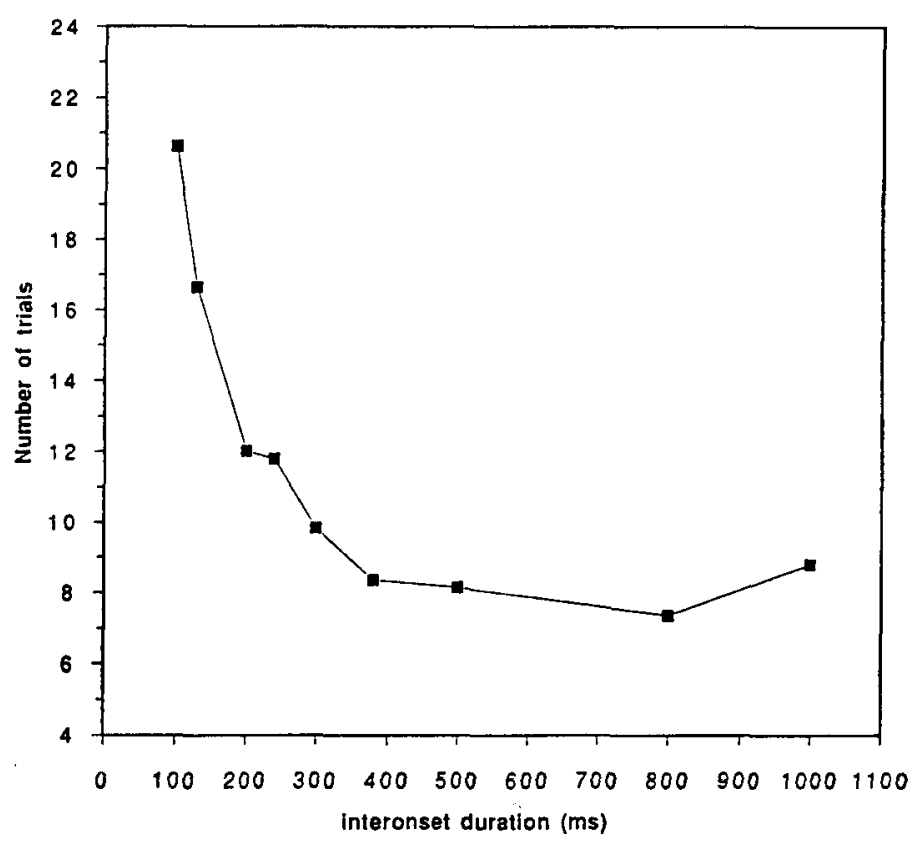

Our results can be compared with those emerging from some previous investigations in Fig. 5. Important to remember here is that in all these investigations different stimuli and different methods were used. Using pairwise presentation of tone sequences Drake $\&$ Botte (in press) measured the tempo discrimination, varying the number of tones in the sequence.

Fig. 5. Comparison with previous work. vanNoorden is the JND for an infinite sequence of notes where every other note was adjusted. Lunney is the JND of every fourth note in an infinite sequence. $D \& B$ $l$ is Drake \& Botte in the case of $J N D$ of single time intervals. $D \& B$ 2 is Drake \& Botte in the case of JND of tempo for regular sequences containing three notes.

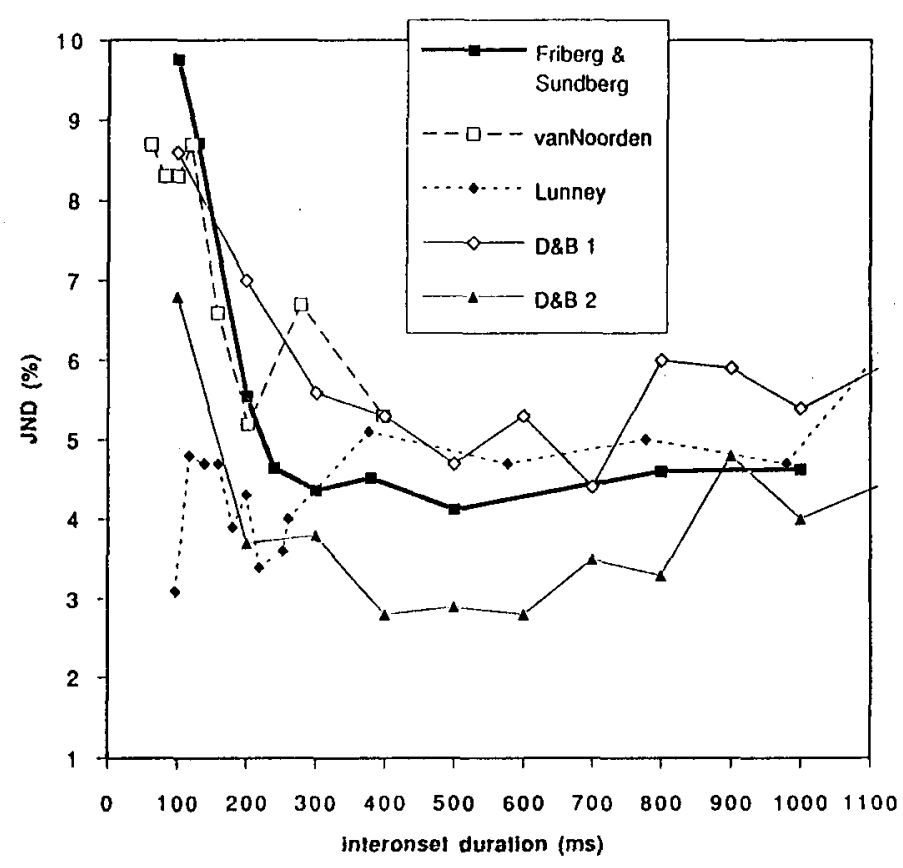


In the figure, DB 1 indicates comparisons of singular intervals and DB 2 comparisons of isochronous sequences containing two time intervals. The overall shape of their curves is quite similar to ours with regard to the increase toward shorter time intervals and relatively constant values for intermediate intervals. However, for longer time intervals the curves of Drake \& Botte show a slight upward slope. Except for short intervals Lunney's (1974) results approach ours quite closely. For shorter time intervals, however, his results seem to deviate from those gained in most other experiments. In the work by van Noorden (1975) about melodic fusion and fission, one of the experiments was comparable to ours; an infinite sequence of tones, where every other tone could be displaced in time.

Comparing the absolute levels of the JND showed that our results were lower than those found for single intervals by Drake \& Botte. On the other hand, they were higher than those found by Drake \& Botte for sequences containing two or more intervals. Unfortunately, both the sounds used for the time markers and the method for determining the JND were different in these two investigations.

Our results showed a reasonably simple dependence on the interonset duration. Therefore, an attempt was made to express the measured JNDs into a piecewise linear approximation. Such an attempt is shown in Fig. 6. There are two line segments, one for intervals shorter than $242 \mathrm{~ms}$ and another one for longer intervals. It is not unlikely that this curve can be used for deriving a method of perceptually uniform perturbation of tone durations in music performance.

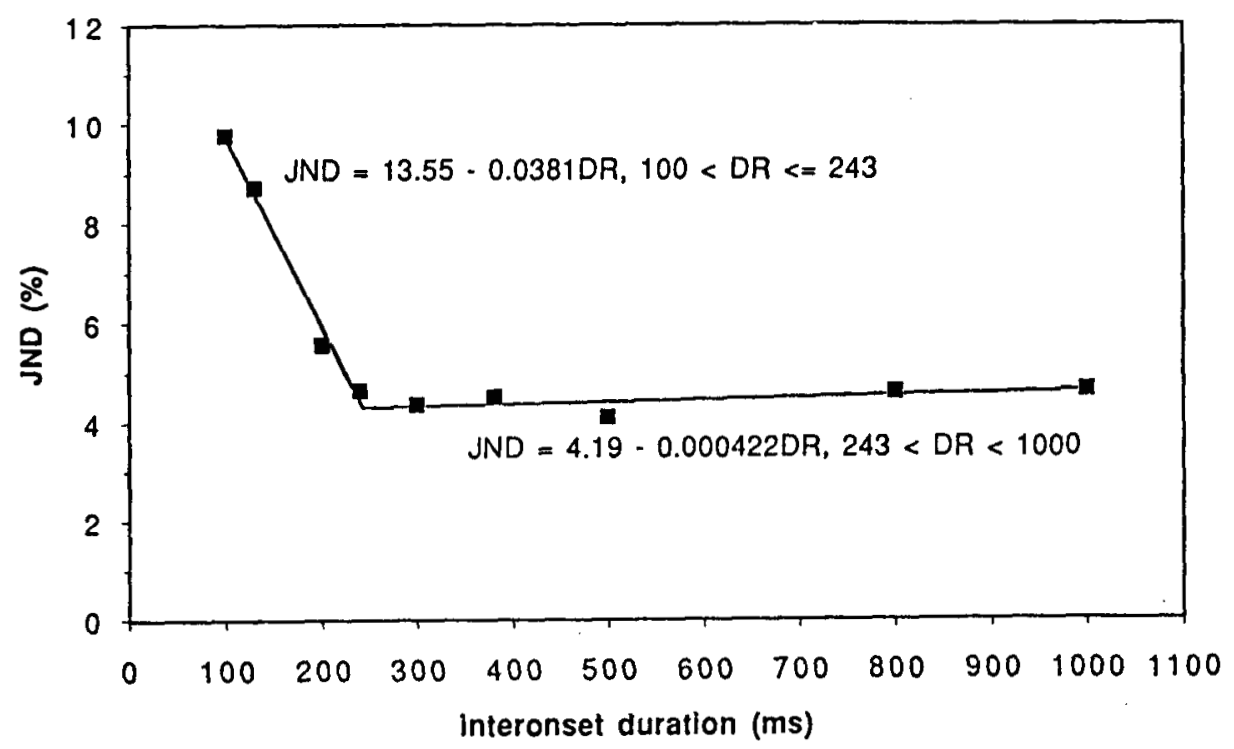

Fig. 6. A two-piece linear approximation of the IND curve. The first line was approximated using the values corresponding to interonset duration $=100 \mathrm{~ms}$ to $240 \mathrm{~ms}$ and the second line with the values corresponding to interonset duration $=300 \mathrm{~ms}$ to $1000 \mathrm{~ms}$.

\section{DISCUSSION}

The effect of musical experience on the detection of small temporal deviations in a music performance have so far yielded contradictory results. In Sundberg et al. (1991a) and Drake \& Botte (in press) there was clear and significant effect of musical experience. On the other hand, Repp (1992b) and this investigation showed little or no effect 
of musical training. Sundberg et al. and Repp both used stimuli close to a real music performance while Drake and this investigation used musically less realistic contexts consisting of short sequences of notes of equal pitch. There can be several possible explanations for the lack of effect of musical experience in our results:

(1) There was too little difference between the groups since almost nobody was a professional musician. However, even if they were not professionals, several subjects had quite extensive musical experience and one was, indeed, professional. Furthermore, a comparison of individual data revealed nothing that suggested that the results of even the most experienced subjects deviated appreciably from those of the rest.

(2) There is a clear difference between listening and performance. Previously it has sometimes been assumed that perception and performance of time are using the same mechanism. However, Sternberg et al. (1982) compared results based on perception and production of fractions of durations, and found diverging results. This supports the hypothesis that perception and performance of timing are in fact different skills.

(3) Perhaps, the skill of perceiving deviations in timing is acquired early or even prenatally. A possible origin of this skill may be speech, where syllable length is an important factor. For instance, the lengthening of a syllable is a very effective way of signalling emphasis, an often essential aspect of the meaning of a sentence.

The knee of the JND curve near $240 \mathrm{~ms}$ is interesting. This duration is close to the average duration of syllables in speech. Also, it is in the vicinity of a typical duration of short tones in musical performance, where lengthening of tone duration is typically used for the purpose of musical expression.

\section{CONCLUSION}

The JND for deviations from a mechanical regularity in a sequence of equal tones plotted as function of tone interonset duration shows a well defined curve. As a rule of thumb, the JND is about $10 \mathrm{~ms}$ for the interonset durations shorter than $250 \mathrm{~ms}$ and about $5 \%$ of the interonset duration for longer durations. No significant effect was found of the subjects' musical experience.

\section{REFERENCES}

Clarke, E., F. (1982): "Timing in the performance of Erik Satie's Vexations", Acta Psychologica 50, pp. 1-19.

Clarke, E., F. (1989): "The perception of expressive timing in music", Psychological Research 51, pp. 2-9.

Drake, C. \& Botte, M.-C. (in press): "Tempo sensitivity in auditory sequences: Implications for the modelling of successive time interval processing" (to appear in Perception and Psychophysics).

Friberg, A. (1991): "Generative rules for music performance: A formal description of a rule system", Computer Music Journal, Vol. 15:2, pp. 56-71.

Gabrielsson, A. and Bengtsson, I. (1983): "Analysis and synthesis of musical rhythm" in Studies of music performance (J. Sundberg, ed.), Stockholm: Royal Swedish Academy of Music, Publication No. 39, pp. 27-60.

Hirsh, I., J., Monahan, C., B., Grant, K., W. and Singh, P., G. (1990): "Studies in auditory timing: 1. Simple patterns", Perception and Psychophysics (47) 3, pp. 215-226 
Lunney, H., W., M. (1974): "Time as heard in speech and music", Nature, Vol. 249, p. 592.

Monahan, C., B., and Hirsh, I. J. (1990): "Studies in auditory timing: 2. Rhythm patterns", Perception and Psychophysics (47) 3, pp. 227-242

Repp, B., K. (1992a): "Diversity and commonality in music performance: An analysis of timing microstructure in Schumann's "Träumerie""', J. Acoust. Soc. Am. 92 (5), pp. 2546-2568.

Repp, B., K. (1992b): "Probing the cognitive representation of musical time: Structural constraints on the perception of timing perturbations", Cognition 44, pp. 241-281.

Schaffer, L. (1981): "Performances of Chopin, Bach and Bartok: studies in motor programming", Cognitive Psychology 13, pp. 326-276.

Sloboda, J., A. (1983): "The communication of musical metre in piano performance", Quarterly Journal of Experimental Psychology 35, pp. 377-396.

Sternberg, S., Knoll, R., L., \& Zukofsky, P. (1982): "Timing by Skilled Musicians" in The Psychology of Music, (D. Deutsch, ed.), New York: Academic Press.

Sundberg, J., Friberg, A., \& Frydén, L. (1991a): "Threshold and preference quantities of rules for music performance", Music Perception Vol. 9, No. 1, pp. 71-92.

Sundberg, J., Friberg, A., and Frydén, L. (1991b): "Common Secrets of Musicians and Listeners - An analysis-by-synthesis Study of Musical Performance" in Representing Musical Structure (P. Howell, R. West \& I. Cross; eds.): London: Academic press.

Todd, N., P. (1985): "A model of expressive timing in tonal music", Music Perception 3, pp. 33-58.

Van Noorden, L., P., A., S. (1975): Temporal coherence in the perception of tone sequences. Eindhoven: Institute for Perception Research. 
